http://jmscr.igmpublication.org/home/ ISSN (e)-2347-176x ISSN (p) 2455-0450 crossref DOI: https://dx.doi.org/10.18535/jmscr/v8i3.78

\title{
A rare case of Rosai-Dorfman disease with sellar, supra sellar involvement and bilateral cavernous sinus extension
}

\author{
Authors \\ Lokeshwar Reddy.C ${ }^{1}$, Einstein Raju ${ }^{2}$, Anand Rajamani ${ }^{3}$, Sofia Bhansal $^{4}$ \\ Department of Radiology, Chettinad Hospital and Research Centre, Kelambakkam, Chennai \\ ${ }^{1}$ Postgraduate, Department of General Medicine, Chettinad Hospital \& Research Institute, Chettinad \\ Academy of Research and Education \\ ${ }^{2}$ Professor, Department of General Medicine, Chettinad, Hospital \& Research Institute, Chettinad Academy \\ of Research and Education \\ ${ }^{3}$ Associate Professor, Department of General Medicine, Chettinad, Hospital \& Research Institute, Chettinad \\ Academy of Research and Education \\ ${ }^{4}$ Postgraduate, Department of General Medicine, Chettinad Hospital \& Research Institute, Chettinad \\ Academy of Research and Education \\ Corresponding Author \\ Dr Lokeshwar Reddy.C
}

\begin{abstract}
Rosai-Dorfman diasease or Sinus histiocytosis with massive lymphadenopathy (SHML) is a disease of unknown etiology. It is a rare non neoplastic histiocytic proliferative disorder that usually presents as bilateral massive lymphadenopathy in cervical regions of neck. It can also involve multiple various extranodal organ systems like eyes, skin, sinuses in less than 30\% of cases. Bone involvement in associated with nodal disease are noted in less than 10\% of cases. A Very few cases have been reported in literature with sellar or suprasellar involvement of Rosai-Dorfman disease. We report a very uncommon case of Rosai-Dorfman disease with sellar, supra sellar involvement and bilateral cavernous sinus extension

Keywords: Rosai-Dorfman disease, sella, supra sella, cavernous sinus.

Key message: sellar and supra sellar involvement with bilateral cavernous sinus extension is very rare.
\end{abstract}

\section{Introduction}

Rosai-Dorfman disease (RDD) or Sinus histiocytosis with massive lymphadenopathy (SHML) is a rare non-neoplastic disease ${ }^{[1]}$. It is a pseudolymphomatous benign disorder and patients usually present with fever, painless bilateral cervical lymphadenopathy, elevated erythrocyte sedimentation rate (ESR), leukocytosis with neutrophilia, and hypergammaglobulinemia ${ }^{[2]}$. Mean onset age of 21 years with $57 \%$ cases reported in men and $43 \%$ cases reported in women ${ }^{[3]}$. Extranodal sites like eyes, skin, sinuses are involved in less than $30 \%$ of cases. Bone involvement in associated with nodal disease are noted in less than $10 \%$ of cases. Less than 10 cases has been reported in literature with sellar or suprasellar involvement of RosaiDorfman disease. 
This case highlights the importance of RosaiDorfman disease as an uncommon but welldocumented differential diagnosis when evaluating sellar or suprasellar regions with massive cervical lymphadenopathy and paranasal sinus involvement.

\section{Case Report}

A 49-year-old south Indian female was admitted with chief complaint of severe head ache, nasal stuffiness and painless cervical lymphadenopathy. Elevated White blood cell count at $15 \mathrm{~K} / \mathrm{microl}$, and C-reactive protein (CRP) and ESR were elevated at $60 \mathrm{mg} / \mathrm{L}$ and $83 \mathrm{~mm} / \mathrm{h}$ respectively at the time of presentation. Her level of Serum uric acid was also elevated to $7.7 \mathrm{mg} / \mathrm{dl}$. Because of severe head ache with no trauma history, Magnetic resonance imaging (MRI) (GE 1.5T) with contrast was recommended further evaluation. The findings were, T2 hypointense homogeneously enhancing lesion with sellar and supra sellar involvement measuring $20 \mathrm{~mm}$ (CC) $\mathrm{x}$ $17 \mathrm{~mm}$ (TS) $\times 5 \mathrm{~mm}$ (AP) noted causing indentation on optic chiasm which is pushed superiorly. There is no significant expansion of sella (figure-1). Enhancing T2 hypointensities were noted in bilateral cavernous sinus encasing internal carotid arteries which were contiguous with and inseperable from above mentioned lesion (figure-2). Enhancing dura is noted in association with bilateral basifrontal lobes in paramedian aspect (figure 4). Nodular enhancement is noted in both paramedian aspects as well. Sub-cutaneous nodules are noted in right molar eminence. Prominent intraparotid nodes are noted. Enlarged parapharyngeal and cervical nodes are noted in visualised region (figure 3 ). Correlative CT showed mixed lytic and sclerotic areas with ground glass matrix noted involving body of sphenoid bone and walls of bilateral ethmoid, maxillary and frontal sinuses. Diffuse opacification of bilateral pan para nasal sinuses was noted.

Based on the imaging findings lymphoma and pseudolymphomatous disease were included in the differential diagnosis. Excisional biopsy of right cervical lymphnode was done. The diagnosis of Rosai-Dorfman disease (RDD) was given based on the pathology report. The histopathology report showed attenuated infiltration of lymphoplasmacytic cells and histiocytes of varying sizes and Foci of emperipolesis within the cellular borders of large, pale histocytic cells. On immunohistochemistry, there was diffuse expression of S-100 protein by the larger histiocytes and all cells were negative for cytokeratin AE1/AE3 and CD1a. Additionally, acid fast bacillus (AFB) and Gomori methenamine silver (GMS) special stains were negative for organisms.

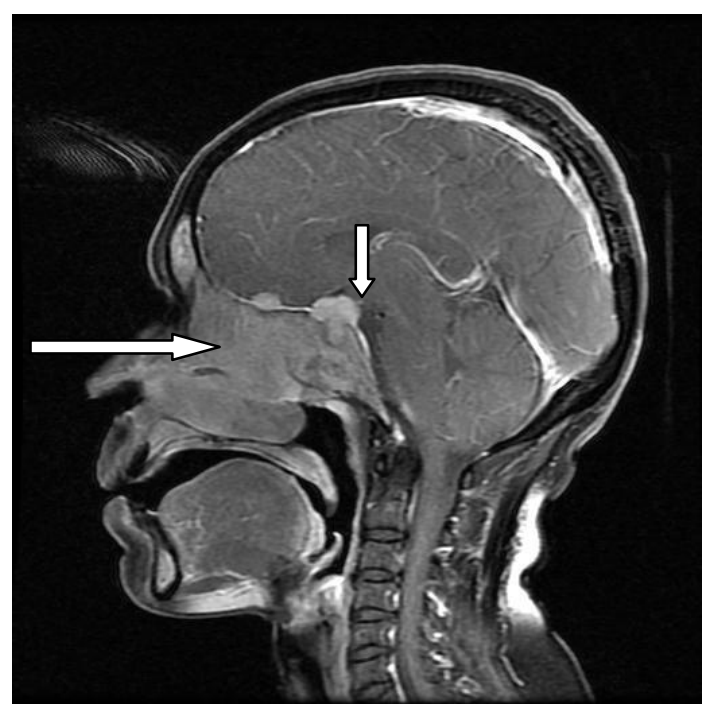

Figure 1- Sagittal T1 Contrast MRI (short arrow shows sellar and supra sellar, dural involvement, long arrow shows paranasal sinuses involvement)

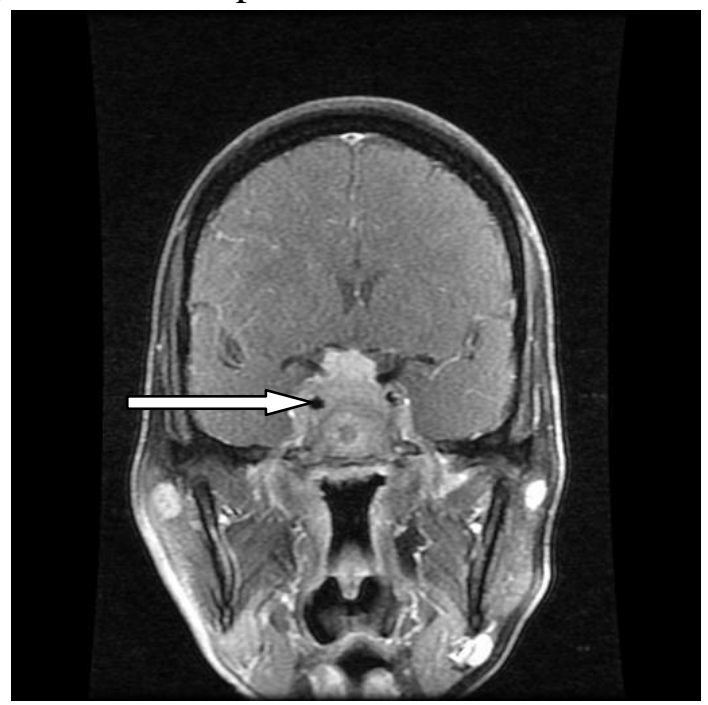

Figure 2- Coronal T1 Contrast MRI (bilateral cavernous involvement by supra-sellar extension) 




Figure 3- Coronal T1 weighted image (Right cervical lymphnode)

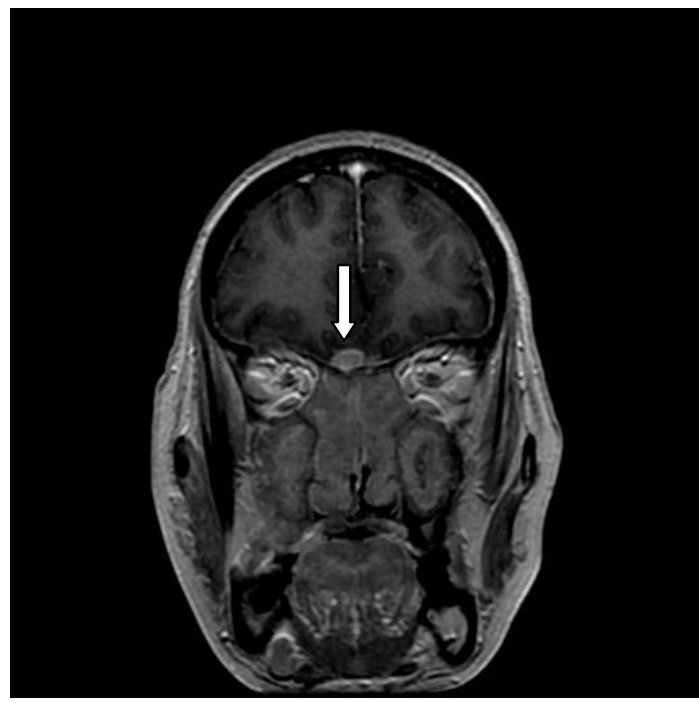

Figure 4- Coronal T1 MRI (Meningeal involvement)

\section{Discussion}

The initial cases of sinus histiocytosis with massive lymphadenopathy were reported by Juan Rosai and Ronald Dorfman ${ }^{[4]}$. Since then, more than 400 cases have been reported ${ }^{[3]}$. In the classic form, RDD presents with painless massive lymphadenopathy, elevated ESR, leukocytosis, hypergammaglobulinemia and anemia. The typical age group affected is from second to third decade of life. There have been reports suggesting delayed initial presentation in later decades of life such as in our patient who was 49 years old.

Extranodal involvement occurs in about less than $30 \%$ cases to sites including orbit, eyelids, upper respiratory tract, salivary gland, skin, testes, and bones ${ }^{[1,3]}$ Osseous involvement occurs in $5-10 \%$ of cases ${ }^{[1,3,5-7]}$.

In 1999 Woodcock RJ et al decribed MR imaging findings in Sinus histiocytosis (Rosai-Dorfman disease) of the suprasellar region ${ }^{[8]}$. In 2010 Wang $F$ et al decribed Intracranial recurrences of Rosai-Dorfman disease in the sellar region in 2 cases $^{[9]}$. In 2017 Huang $N$ etal described 7 cases of Extra-nodal Rosai-Dorfman disease in head and neck. 3 out of seven cases had sellar region involvement ${ }^{[10]}$. In our patient there are multiple regions of involvement including sellar, supra sellar regions with contiguous bilateral cavernous sinuses that is very rare. There were also enhancing meningeal lesions, hyperostosis of paranasal sinuses with mucosal proliferation, subcutaneous nodules in right maxillary region and bilateral cervical lymphadenopathy.

In summary, RDD is a rare disease of marrow hematopoietic stem cell origin, with painless massive lymphadenopathy being the classic finding. However, less than $30 \%$ of patients have extranodal presentation with less than $10 \%$ showing pure osseous involvement. A Very few cases has been reported in literature with sellar or suprasellar involvement of Rosai-Dorfman disease. S-100 positive histiocytes along with emperipolesis (inflammatory cells "passing through" the cytoplasm of the large histiocytes) on biopsy confirms the diagnosis.

\section{Conclusion}

This case was reported as it was a rare disease and sellar/supra sellar involvement with bilateral cavernous sinus extension was even rarer. As the disease generally resolves spontaneously, the patient is currently on observation with steroids only treatment.

\section{References}

1. Demicco EG, Rosenberg AE, Björnsson J, Rybak LD, Unni KK, Nielsen GP. Primary Rosai-Dorfman disease of bone: A 
clinicopathologic study of 15 cases. Am J

Surg Pathol 2010.

2. Rosai J, Dorfman RF. Sinus histiocytosis with massivelymphadenopathy: A pseudolymphomatous benign disorder. Analysis of 34 cases. Cancer 1972.

3. Foucar E, Rosai J, Dorfman R. Sinus histiocytosis with massive lymphadenopathy (Rosai-Dorfman disease): Review of the entity. Semin Diagn Pathol 1990.

4. Rosai J, Dorfman RF. Sinus histiocytosis with massive lymphadenopathy. A newly recognized benign clinicopathological entity. Arch Pathol 1969.

5. Walczak BE, Halperin DM, Bdeir RW, Irwin RB. Orthopaedic case of the month: A 50-year-old woman with persistent knee pain. Clin Orthop Relat Res 2011.

6. Dean EM, Wittig JC, Vilalobos C, Garcia RA. A 16-year-old boy with multifocal, painless osseous lesions. Clin Orthop Relat Res 2012.

7. Loh SY, Tan KB, Wong YS, Lee YS. Rosai-Dorfman disease of the triquetrum without lymphadenopathy. A case report. J Bone Joint Surg Am 2004.

8. Woodcock RJ, Mandell JW, Lipper MH. Sinus histiocytosis (Rosai-Dorfman disease) of the suprasellar region: MR imaging findings - A case report. Radiology. 1999.

9. Wang F, Qiao G, Lou X, Song X, Chen W. Intracranial recurrences of Rosai-Dorfman disease in the sellar region: Two illustrative cases. Acta Neurochir (Wien). 2011.

10. Huang N, Cao D, Chen T, Lin N, Wu Y, Zeng Z. Extra-nodal Rosai-Dorfman disease in head and neck: Seven cases report and literature review. Chinese Journal of Medical Imaging Technology. 2017. 